\title{
La Amiodarona reduce la mortalidad tanto en la insuficiencia cardíaca como en el post-infarto de miocardio
}

\author{
Effect of prophylactic amiodarone on mortality after acute myocardial infarction and in congestive heart failure: \\ meta-analysis of individual data from 6500 patients in randomised trials. \\ Amiodarone Trials Meta-Analysis Investigators. Writing committee: Connolly S, Cairns J, Gent M, et. al. Lancet 1997; 350:1417-24.
}

\section{Objetivo}

Valorar los riesgos y beneficios de la amiodarona profiláctica en pacientes de alto riesgo arrítmico post-Infarto Agudo de Miocardio (post-IAM) o con Insuficiencia Cardíaca Congestiva (ICC).

\section{Fuente de Datos y Selección de Estudios}

Se incluyeron estudios randomizados que compararan amiodarona contra placebo o tratamiento usual.

\section{Extracción de Datos}

Los datos individuales de los pacientes se extractaron acorde a un protocolo predefinido por los principales investigadores de los estudios seleccionados.

\section{Resultados principales}

Se seleccionaron 8 ensayos en pacientes post-IAM reciente ( 6 dentro del mes y 2 dentro de los 2 meses) y 5 de ICC. Nueve estudios eran doble-ciego, con placebo y 4 comparaban amiodarona con tratamiento usual. De los 6553 pacientes, $78 \%$ provenían de los estudios post-IAM (seleccionados, excepto en 2 estudios, como pacientes de alto riesgo por Holter y ECG o fracción de eyección [FE] anormales). El otro $22 \%$ provenían de los estudios de ICC (criterio de alto riesgo por FE y en 2 además por Holter). El seguimiento fue de 0.4 a 2.5 años. El $89 \%$ había tenido un IAM previo. La mortalidad total se redujo 13\%, OR 0.87 (IC 95\% 0.78-0.99, p=0.03). La muerte de causa arrítmica se redujo $29 \%$ 0R 0.71 (IC $95 \% 0.59-0.85, p=0.0003$ ) y no hubo efecto sobre las muertes no-arrítmicas. Los efectos benéficos persisten en el tiempo. No hubo diferencias en el beneficio entre los estudios post-IAM y los de ICC. El riesgo de muerte arrítimica en el grupo control fue mayor en los estudios de ICC $(10.7 \%)$ que en los de post-IAM (4.1\%), siendo la ICC (clase funcional 3-4 de la NY Heart Association) el mejor predictor individual de muerte arrítimica. En los estudios doble ciego, los pacientes asignados a amiodarona abandonaron un 14\% más que el grupo placebo. El hipotiroidismo fue la causa más común y las alteraciones hepáticas raramente requirieron suspensión. El exceso de riesgo de toxicidad pulmonar fue del $1 \%$ por año.

\section{Conclusiones}

La amiodarona profiláctica en pacientes de alto riesgo post-IAM o con ICC reduce la tasa de muerte arrítimica y este efecto redunda en un $13 \%$ de reducción global de la mortalidad total.

\section{COMENTARIO}

A pesar de los avances terapéuticos, la mortalidad al año entre los supervivientes post-IAM continúa siendo $>10 \% \mathrm{y}$, al igual que en los pacientes con ICC, es debido principalmente a la muerte arrítmica-súbita (1). Con excepción de los ß-bloqueantes ninguna droga antiarrítmica ha mostrado reducir la mortalidad y en algunos casos la ha incrementado (inclusive el sotalol) (2). La principal ventaja de este estudio es que, a diferencia de los estudios individuales, logra un número de pacientes suficiente (y por ende un gran poder estadístico) para detectar confiablemente reducciones del 10-20\% en la mortalidad.

Dado que muchos estudios no hallaban reducción de la mortalidad total, a pesar de reducir la muerte arrítmica, fue de especial interés saber que la amiodarona no aumentó las muertes no-arrítmicas ( $57 \%$ del total). La reducción del $13 \%$ de la mortalidad total hallada en este meta-análisis parece clínicamente importante pero, dado que esta estimación todavía tiene una considerable variabilidad (entre un 1 y un $22 \%$ de reducción), deberíamos seguir acumulando evidencia a través nuevos ensayos clínicos para definir más adecuadamente el rol de la amiodarona en este tipo de pacientes.

El análisis de eficacia (sólo de los pacientes que recibieron tratamiento con amiodarona) mostró reducciones altamente significati- vas del $18 \%$ de la mortalidad total y $35 \%$ de muerte súbita arrítmica. El análisis de subgrupos sólo halló diferencias significativas en la eficacia de la amiodarona entre los estudios controlados con placebo y los que comparaban con tratamiento usual en cuanto a mortalidad total. La mayor eficacia observada en los estudios abiertos*, a diferencia de los estudios ciegos *, podría explicarse por un seguimiento más intensivo o la menor tasa de suspensión de la amiodarona. Sin embargo no hubo diferencias con la muerte arrítmica. Tampoco hubo diferencias de efectividad entre pacientes post-IAM y aquellos con ICC, ni se vio influenciada por la FE, la clase funcional o la presencia de arritmias ventriculares asintomáticas en el Holter, lo que la hace aplicable a un amplio espectro de pacientes. ¿Deberíamos usar amiodarona profilácticamente en pacientes postIAM y en pacientes con ICC? Aunque el beneficio relativo es similar entre diferentes tipos de pacientes el beneficio absoluto es indudablemente mayor entre los pacientes de más alto riesgo de muerte arrítmica (ICC o severa disfunción sistólica). Como ejemplo; en clase funcional 3-4 (12.2\% de riesgo anual de muerte arrítmica) tratar 100 pacientes/año prevendría 3.5 muertes. Por lo tanto la amiodarona profiláctica sería razonable, por ahora, en pacientes con riesgo particularmente alto de muerte por arritmias.

\section{Referencias}

1. LeFeuvre CA, Connolly SJ, Cairns JA, et al. Comparison of mortality from acute myocardial infarction between 1979 and 1992 in a geographically-defined stable population. Am J Cardiol 1996; 78:1345-49.

2. Waldo AL, Camm AJ, de Ruyter H, et al. Effect of d-sotalol on mortality in patients with left ventricular dysfunction after recent and remote myocardial infarction. Lancet $1996 \cdot 3458 \cdot 7-12$ 